\title{
Pressure induced phase transitions in Germanium Telluride: Raman signatures of anharmonicity and oxidation
}

\author{
Amit Pawbake, ${ }^{1, *}$ Christophe Bellin, ${ }^{1}$ Lorenzo Paulatto, ${ }^{1}$ Keevin Béneut, ${ }^{1}$ Johan \\ Biscaras, ${ }^{1}$ Chandrabhas Narayana, ${ }^{2}$ Dattatraya J. Late, ${ }^{3}$ and Abhay Shukla ${ }^{1, \dagger}$ \\ ${ }^{1}$ Institut de Minéralogie, de Physique des Matériaux et de Cosmochimie, \\ Sorbonne Université, UMR CNRS 7590, MNHN, \\ IRD UMR 206, 4 Place Jussieu, F-75005 Paris, France \\ ${ }^{2}$ Chemistry and Physics of Materials Unit, \\ Jawaharlal Nehru Centre for Advanced Scientific \\ Research, Jakkur, Bangalore 560 064, India \\ ${ }^{3}$ Physical and Materials Chemistry Division, \\ CSIR-National Chemical Laboratory, \\ Dr. Homi Bhabha Road, Pune 411008, India
}

\begin{abstract}
Pressure induced phase transitions in GeTe, a prototype phase change material have been studied to date with diffraction which is not sensitive to anharmonicity induced dynamical effects. GeTe is also prone to surface oxidation which may compromise surface sensitive measurements. These factors could be responsible for the lack of clarity about the phases and transitions intervening in the phase diagram of GeTe. We have used high pressure Raman scattering and $a b$ initio pseudopotential density functional calculations to unambiguously establish the high pressure phase diagram and identify three phases up to $57 \mathrm{GPa}$, a low-pressure rhombohedral phase, an intermediate pressure cubic phase and a high pressure orthorhombic phase. We detect substantial broadening and softening of Raman modes at low pressure and identify the transition regions and possible intermediate phases.
\end{abstract}

DOI:

PACS numbers: 
Rewritable optical technology used in CDs and DVDs is based on the existence of two solid phases, crystalline and amorphous, of phase change materials $[1,2]$. Phase change materials may also have more than one crystalline phase as in the prototype material GeTe which exists in the rhombohedral phase $(R 3 m)$ at room temperature and adopts the $\mathrm{NaCl}$ structure $(F m \overline{3} m)$ above $700 \mathrm{~K}$. The rhombohedral phase is a distortion of this cubic structure along the [111] direction. The different phases and the mechanisms governing the phase transitions are of great interest, driven by the possibility of optimizing materials and their properties for new applications. Phase transitions can also be provoked by applying pressure. In GeTe, the ambient pressure rhombohedral phase is thought to transit to a cubic $\mathrm{NaCl}$ structure with further transitions within a range of a few tens of GPa. Since a metastable cubic $\mathrm{NaCl}$ phase is also found in ambient conditions and the transition between this phase and an amorphous phase is technologically relevant, investigating pressure induced phase transitions assumes importance. GeTe is also used in prototype devices, in the form of nanoparticles or nanowires [3, 4] and in this form it is particularly sensitive to oxidation which alters its properties. In this work we establish the pressure phase diagram of GeTe by high pressure Raman scattering and ab initio calculations. We unambiguously determine all phases and underlying mechanisms of the transitions up to $57 \mathrm{GPa}$. We show that care has to be taken to avoid surface oxidation contamination, especially in Raman studies.

While studying the temperature driven rhombohedral to cubic phase transition, Chattopadhyaya et al. [5] remarked that the cubic phase is also obtained at room temperature but at higher pressure. They speculated that as in IV-VI compounds like SnTe or PbTe, orthorhombic and $\mathrm{CsCl}$ type cubic phases may be found at still higher pressures. Reasons for this polymorphism in IV-VI compounds were addressed in earlier works $[6,7]$, where the lower pressure rhomobohedral-cubic transition was associated with anharmonicity and the higher pressure cubic-orthorhombic transition was understood in the context of increasing covalent nature of bonds. Subsequent work in GeTe has concentrated on X-ray diffraction studies of pressure induced phase transitions. Redon and Leger [8] found that the rhombohedral to cubic transition occurs at $5 \mathrm{GPa}$ in non-hydrostatic conditions and only beyond $8 \mathrm{GPa}$ in hydrostatic conditions. They also detected an orthorhombic phase along with the cubic phase in a possible mixed phase scenario but no other transitions up to the highest measured pressure of $25 \mathrm{GPa}$. Serebryanaya et al. [9] found that the cubic $\mathrm{NaCl}$ phase exists alone till at least $19 \mathrm{GPa}$ and that at higher pressure shear deformation or non-hydrostatic 
conditions generate an orthorhombic phase and the $\mathrm{CsCl}$ phase. They also found that the $\mathrm{CsCl}$ cubic phase appears in hydrostatic conditions at $43 \mathrm{GPa}$. Meanwhile Onodera et al. [10] found the rhombohedral to cubic transition at $3 \mathrm{GPa}$, and an orthorhombic phase at 18 Gpa. These results lack consistency.

Non-hydrostatic conditions and the propensity of GeTe for shear deformations may be the reason for this lack of consistency. Another preponderant effect in this material is anharmonicity [11], treated in theoretical models for the temperature driven phase transition [12]. Anharmonicity is crucial for all prospective thermoelectric applications [13]. Several authors have also simulated pressure driven phase transitions. Ciucivara et al. [14] found a first order phase transition but with a small volume discontinuity at 5.4 GPa. They predicted that the transition pressure was close to the semiconductor-metal transition pressure. This was confirmed by Do et al. [15] who predict a semiconductor to metal transition at $6 \mathrm{GPa}$, in the $\mathrm{NaCl}$ phase. Their work indicates that though this low temperature phase transition is relatively straightforward, at pressures between 20-30 GPa, GeTe may exist in several phases. Finally Sun et al. [16] performed ab initio molecular dynamics calculations and analysed bond strengths across different phases. They concluded that the phase transitions are reversible and that there is indeed a semiconductor metal transition beyond the rhombohedral phase. They explain these transitions by a Peierls mechanism which distorts crystal structure and creates an electronic gap at low pressure. Recent work [17-19] has identified GeTe and other phase change materials as belonging to a class of materials defined as incipient metals with 'metavalent' chemical bonding which is neither covalent nor metallic and accounts for a wide variety of phenomena including polymorphism, anharmonicity and Peierls distortions.

Polarized Raman measurements of a GeTe single crystal (from 2D semiconductors) revealed that the surface is sensitive to oxidation through exposure to ambient conditions or to solvents like ethanol. A freshly cleaved surface however is representative of pristine GeTe and is preserved over the time necessary for loading the sample in the pressure cell. The sample was loaded in a membrane diamond anvil cell (DAC) [20] using a stainless steel gasket with a $250 \mathrm{~mm}$ diamond culet and neon as the pressure transmitting medium [21]. The R1-line emission of a tiny ruby [22] was used for pressure calibration [23]. The high pressure, 300K Raman experiments used a Jobin-Yvon HR-460 spectrometer (1500 gratings/mm monochromator and Andor CCD camera) in backscattering with a $514.5 \mathrm{~nm} \mathrm{Ar}$ 
laser focused to a $2 \mu \mathrm{m}$ spot with incident power on the DAC limited below $2 \mathrm{~mW}$. Three volume Bragg filters ensured Rayleigh rejection and the $12 \mathrm{~cm}^{-1}$ low frequency cut-off. A remnant low energy tail was subtracted using a polynomial background. The high pressure data, acquired from 120 to 900 seconds per pressure point depending on count rate was binned by a factor of 5 to increase statistics.

Raman scattering has been widely used to study GeTe. GeTe has four Raman active modes [24], the $A_{1}$ and the $E$ modes with longitudinal and transverse symmetry. However real crystals have a large number of Ge vacancies which are self-doping [25], leading to screening of long range interactions. Thus two modes are found at the zone center [26, 27] due to degeneracy of long wavelength longitudinal and transverse modes. In backscattering, with the sample $(a, b)$ plane perpendicular to the laser light these two modes should be visible in an unpolarised or parallel polarization measurement. In existing Raman measurements there is a lack of clarity about the measured Raman active modes. Many experimental studies show two modes around 90 and $120 \mathrm{~cm}^{-1}$ or two modes around 120 and $140 \mathrm{~cm}^{-1}$ or even three modes around 90, 120 and $140 \mathrm{~cm}^{-1}$. The mode at about $140 \mathrm{~cm}^{-1}$ is often identified as an intrinsic GeTe mode [3, 28-30]. In Fig.1, by comparing a freshly cleaved surface to a surface exposed to ambient conditions we show that this mode is due to surface oxidation and also obtained if a freshly cleaved surface is washed with ethanol. Oxidation partially or completely suppresses the lower energy mode $\left(90 \mathrm{~cm}^{-1}\right)$ and gives rise to the spurious oxide mode $\left(140 \mathrm{~cm}^{-1}\right)$. In the inset of Fig. 1 we show polarization analysis of the Raman spectrum. Both peaks are visible in back scattering in the unpolarised or parallel polarization measurement whereas in a crossed polarization measurement the lower energy $E$ mode is visible while the higher energy $A_{1}$ mode is suppressed. We add that the spurious peak is insensitive to polarisation analysis. Another indication of oxidation is that it leads to narrowing of the Raman peaks [31]. Intrinsic Raman peaks at ambient and low pressure in GeTe are considerably broadened, with a full width at half maximum beyond $20 \mathrm{~cm}^{-1}$. This is due to the anharmonic nature of GeTe optical phonons, also responsible for the high temperature transformation to the cubic phase. Anharmonicity should also reveal itself through anomalous softening of Raman modes with pressure. This is the motivation to perform high pressure Raman experiments. Our pressure medium neon allows for quasihydrostatic conditions in the measured pressure range and lacks Raman activity thus being a better choice than liquid (ethanol/methanol mixtures) or solid media used for pressure 


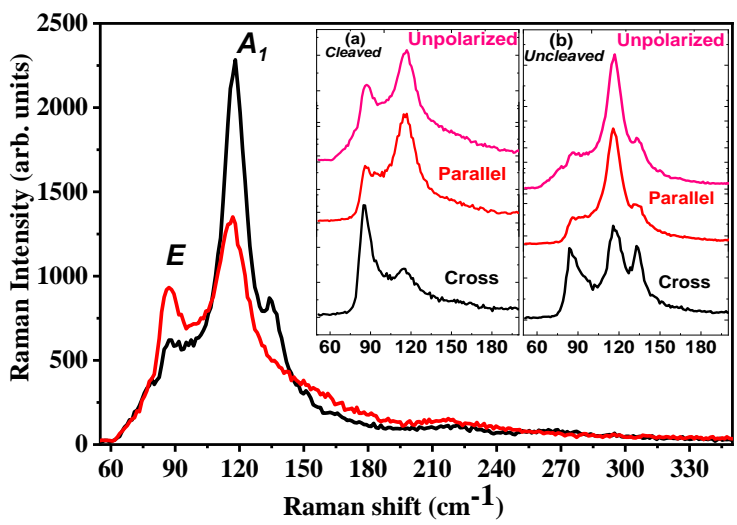

FIG. 1. Raman spectra of freshly cleaved sample (red line) and a surface exposed to ambient conditions (black line), showing the effect of oxidation. The inset shows the polarization analysis of these features, the $A_{1}$ peak being suppressed in crossed polarization.

transmission in all earlier experiments. Importantly neon does not react with the GeTe surface. As we have seen in Fig.1, exposure to alcohol leads to oxidation of the surface compromising the Raman experiment which is surface sensitive. Diffraction experiments are bulk sensitive but when the sample is in powder form with small grain size surface oxidation could still be a problem.

In Fig.2 we show the measured Raman spectra from ambient pressure to 57 GPa. Visually, three regions corresponding to different Raman spectra can be immediately identified, from ambient pressure to $4 \mathrm{GPa}, 4-15 \mathrm{GPa}$ and 15-57 GPa. These three regions are confirmed by analysis of the peak positions of Fig.2 as shown in Fig.3. In the low pressure region two broad peaks are seen corresponding to the $R 3 m$ rhombohedral phase as confirmed in the polarized measurements. Their relative intensities can vary from sample to sample. These peaks broaden even more on the application of pressure and soften to finally disappear above $4 \mathrm{GPa}$. The $E$ mode broadens from 21 to $34 \mathrm{~cm}^{-1}$ while the $A_{1}$ mode broadens from 25 to more than $60 \mathrm{~cm}^{-1}$. Both broadening and softening underline the role of anharmonicity in driving the low pressure phase transformation which is signalled by a low energy mode close to the transition pressure.

As noted above, some calculations $[14,16]$ predict an insulator-metal transition corresponding to the cubic phase. Our experiments cannot detect this eventuality, but in this case the peak broadening at the transition could also correspond to enhanced electronphonon coupling. Between 4 and 15 Gpa, we do not measure significant Raman intensity. 


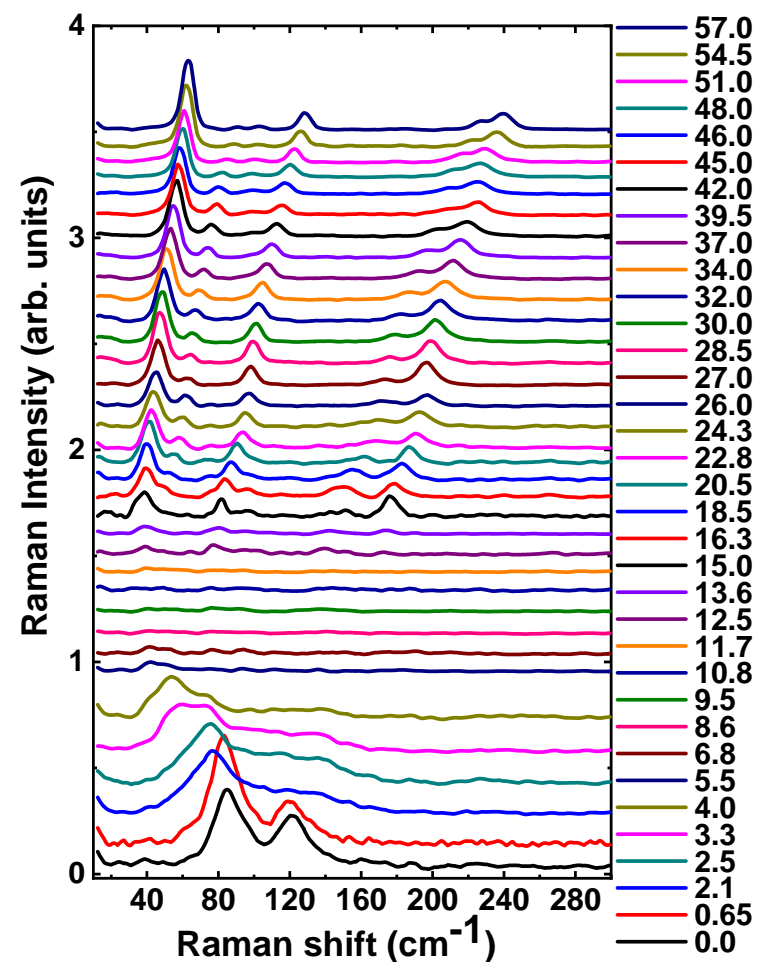

FIG. 2. Raman spectra of GeTe from ambient pressure to $57 \mathrm{GPa}$, in quasi hydrostatic conditions. Three regions of distinct Raman signals can be visually identified between ambient pressure and $4 \mathrm{GPa}, 4-15 \mathrm{GPa}$ and 15-57 GPa. The low pressure region is characterized by broad peaks and softening with pressure. A non-dispersing feature around $100 \mathrm{~cm}^{-1}$ with very low intensity is identified as a parasite. All pressure values are in GPa.

This absence is coherent with the cubic $\mathrm{NaCl}$ structure which does not have Raman active modes. Between 3 and $6 \mathrm{GPa}$, an intense mode with a weak shoulder is seen around 50 $\mathrm{cm}^{-1}$. Between 12 and $15 \mathrm{GPa}$ a few very low intensity modes can be identified. Both these regions possibly correspond to mixed phases as discussed below. Above 15 GPa most of the modes appearing in the 12-15 GPa region reinforce themselves as the signature of the third phase. We identify three low energy modes dispersing linearly between 40 and $130 \mathrm{~cm}^{-1}$ and two modes at higher energy dispersing linearly between 150 and $240 \mathrm{~cm}^{-1}$ in the pressure range 15-57 GPa. These transitions can also be clearly seen in Fig.3.

Earlier diffraction experiments indicated the existence of orthorhombic, cubic $\mathrm{CsCl}$ or monoclinic phases or eventual co-existence of these. No consistency was found as to the transition pressure. Various phases and transition pressures were also predicted in calculations. We therefore performed total energy and phonon ab initio pseudopotential density functional 


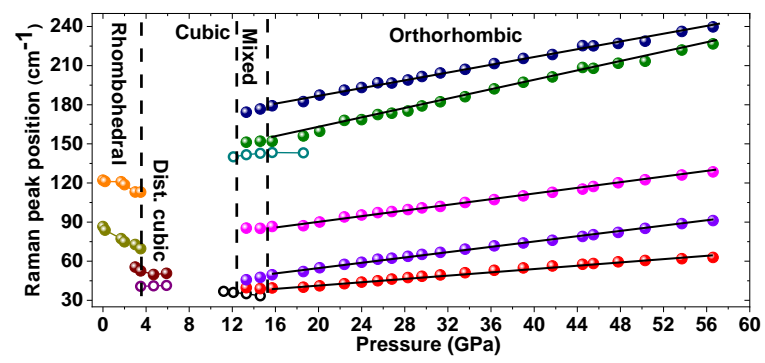

FIG. 3. Pressure dependence of Raman modes from ambient pressure to $57 \mathrm{GPa}$ for a freshly cleaved sample as determined from the data of Fig.2, showing the phase transition regions and the three phases. Full circles indicate modes with significant intensity while empty circles indicate modes with weak intensity, present in the vicinity of transitions.

calculations at about a hundred different unit cell volumes using the Quantum-ESPRESSO $[32,33]$ code with SG15-ONCV pseudopotentials [34, 35] and the Perdew-Burke-Ernzerhof functional [36] with Grimme-D2 van der Waals correction [37] for five different structures. In Fig. 4 we show the variation with pressure of Raman mode energies for the rhombohedral $(R 3 m)$, cubic $(F m \overline{3} m)$, distorted cubic (R3m which is a cubic distortion along the [111] direction) and orthorhombic (Pnma and Cnmn) unit cells. The monoclinic structure was not found to be relevant both from the point of view of stability and the possible Raman modes involved. For each volume the unit cell (rhombohedral and orthorhombic) and internal degrees of freedom (distorted cubic, rhombohedral and orthorhombic) were relaxed while keeping the initial crystal symmetry. To ensure consistent and smooth variation of the calculated quantities we fixed the Fourier transform grid used to represent the wavefunctions. It was determined for the largest volume using a kinetic energy cutoff of $60 \mathrm{Ry}$ and further incremented to account for anisotropic cell relaxation for the orthorhombic unit cell. The evolution of the total energy as a function of the volume was fitted with a Birch first order equation of state (EOS). From the EOS we obtain the enthalpy as a function of the pressure. Below $40 \mathrm{GPa}$ the pressure found from the EOS and from the ab initio calculation is consistent within 5\% and 1 Gpa. By symmetry analysis we determined Raman active modes at the final relaxed coordinates, allowing some tolerance in the determination of symmetry of the relaxed structure.

In the low pressure region the structure compatible with our measurements is the rhombohedral one (Fig.4(c)) with two modes that anomalously soften with pressure. In the 


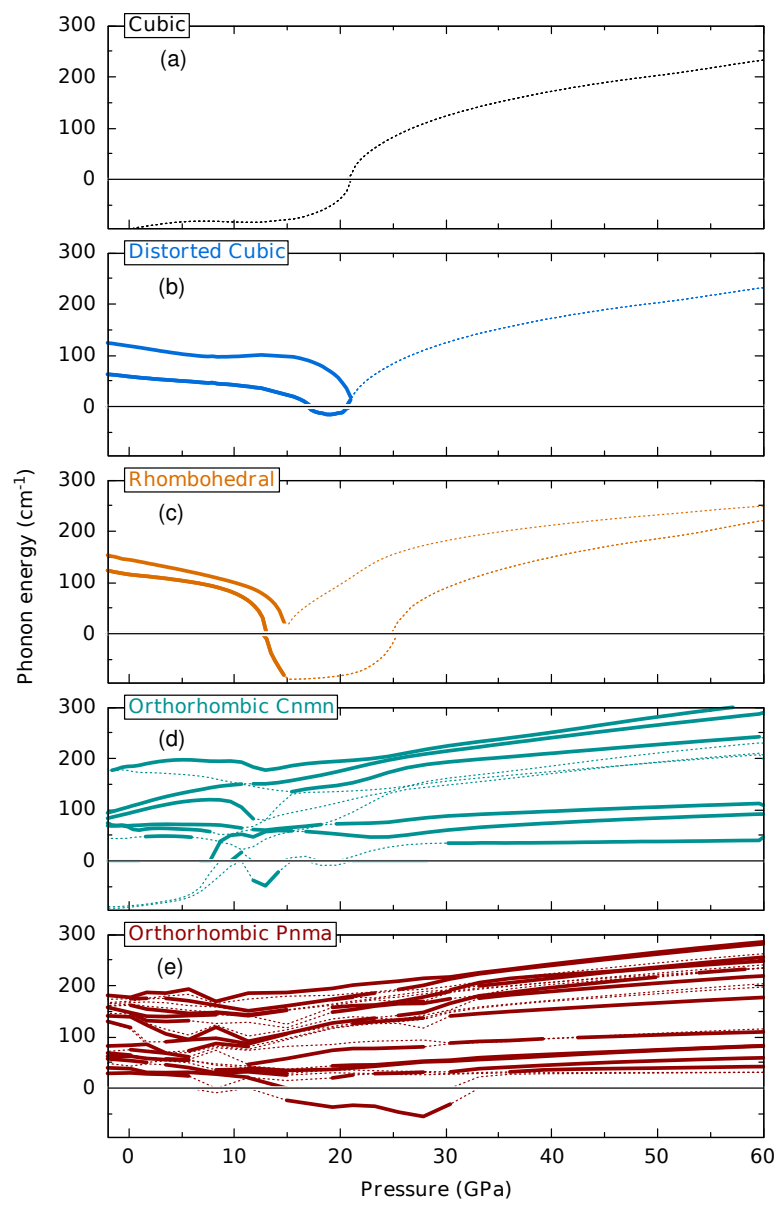

FIG. 4. Calculation of Raman modes for various possible phases as a function of pressure. Thick lines indicate Raman active modes, dashed lines indicate Raman inactive modes. Negative frequencies indicate unstable modes. (a) cubic (b) distorted cubic (c) rhomboherdral (d) orthorhombic (Cnmn) (e) orthorhombic (Pnma).

intermediate pressure range with a vanishing Raman signal, the cubic unit cell $(\mathrm{NaCl})$ with no Raman active modes is the only compatible structure (Fig.4(a)). At the transition from rhombohedral to cubic we detect a strong mode with a shoulder around $50 \mathrm{~cm}^{-1}$, over a small pressure range (3-6 GPa). By inspection of Fig.4(b) this probably corresponds to the low energy mode of the distorted cubic structure. From the transition at 15 GPa to 57 GPa we measure three low energy modes and two higher energy modes. Fig. 4(d) and 4(e) show that the orthorhombic unit cells are compatible with these modes as there is close correspondence with the experiment as seen in Fig.3. We note that only the orhtorhombic structure has low lying modes below $130 \mathrm{~cm}^{-1}$ in the measured pressure range and this phase remains unchanged till $57 \mathrm{GPa}$. The three low energy modes are resolution limited in width. 

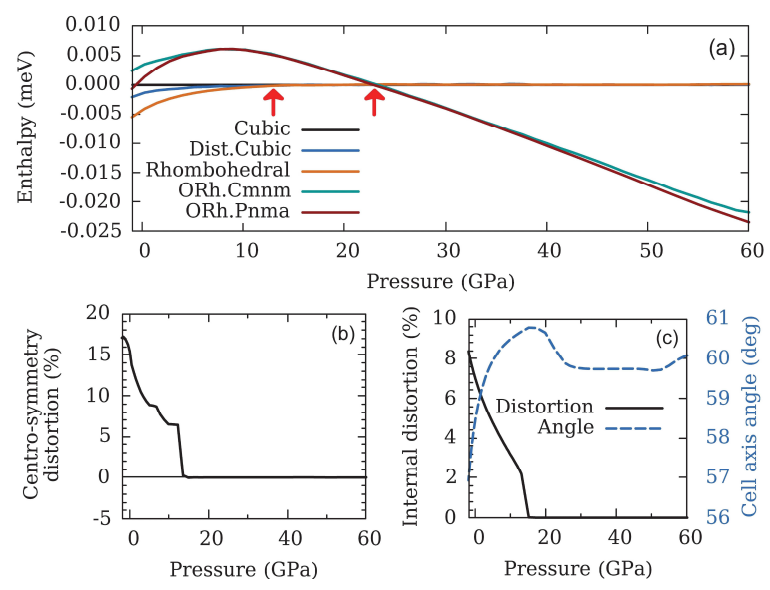

FIG. 5. (a) Stability of the phases of Fig.4, as a function of pressure as predicted by our calculation with transition pressures shown by red arrows. The enthalpy is shown with reference to the cubic phase enthalpy(zero base line). (b) Deviation of Ge atoms in the Pnma structure from the symmetry center in the Cnmn structure. (c) Rhombohedral to cubic transformation as a function of pressure with the percentage deformation of the internal degree of freedom (black line, left axis) and the angle between unit cell vectors (blue dashed line, right axis).

The two weaker modes at higher energy are broader, but this could also be a result of nearly degenerate modes.

In Fig.5(a), we show the enthalpy for the various structures with reference to the enthalpy of the cubic phase, as a function of pressure. The calculation correctly predicts all the phases and transitions: rhombohedral to cubic and cubic to orthorhombic. The transition pressures are typically higher with respect to experiment by 8-10 GPa. This may be due to higher order effects like anharmonicity not sufficiently accounted for in the calculation. The Pnma and Cnmn structures are very similar, the only difference being that the first is not centro-symmetric. In Fig.5(b), we trace the deviation of the Ge atoms in the Pnma structure from the centro-symmetric structure and find that above $15 \mathrm{GPa}$, where they are relevant in the phase diagram the two structures become identical, the residual discrepancy in the enthalpy being explained by residual numerical error. Interestingly at low pressure the distorted cubic phase lies energetically between the rhombohedral and the cubic phase, implying that it characterizes the transition path between these two phases. In effect this phase is predicted to have a low energy Raman active mode around $50 \mathrm{~cm}^{-1}$ (Fig.4(b), seen over the range of 3-6 GPa in our data. In Fig.5(c), we show how the rhombohedral unit cell 
transforms to the cubic unit cell as pressure increases. At high pressure, the orthorhombic phase becomes energetically favoured as clearly seen in our Raman data.

In this work we use high pressure Raman scattering and ab initio pseudopotential density functional calculations to establish the pressure phase diagram of GeTe to 57 GPa. Raman scattering is sensitive to subtle structural changes and is an ideal tool for highlighting the role of a dynamical effect like anharmonicity, important in the low pressure phase. However care must be taken to avoid oxidized surfaces which can compromise Raman measurements. We detect substantial anharmonic broadening and anomalous softening of Raman modes on the application of pressure leading to the low pressure rhombohedral to cubic transition mediated by a distorted cubic phase. We also clearly establish that the high pressure phase is orthorhombic and that both transitions are accompanied by regions of mixed phases.

A.P. acknowledges the CEFIPRA for Raman-Charpak fellowship 2016 and IMPMC for hosting.

* tamitpawbake@gmail.com

† abhay.shukla@sorbonne-universite.fr

[1] S. Raoux, ,W. Welnic, and D. Ielmini, Chem. Rev. 110, 240 (2009)

[2] M. Wuttig and M. Yamada, Nat. Mater. 6, 824 (2007)

[3] M. J. Polking, J. J. Urban, D. J. Milliron, H. Zheng, E. Chan, M. A. Caldwell, S. Raoux, C. F. Kisielowski, J. W. Ager, III, R. Ramesh, and A. P. Alivisatos, Nano Lett. 11, 1147 (2011)

[4] S. Park, D. Park, K. Jeong,T. Kim, S. Park, M. Ahn, W. J.Yang, J. H.Han, H. S.Jeong, S. G. Jeon, and J. Y. Song, ACS Appl. Mater. Interfaces. 7, 21819 (2015)

[5] T. Chattopadhyay and J. X. Boucherle, J. Phys. C: Solid State Phys. 20, 1431 (1987)

[6] P.B. Littlewood, J. Phys. C: Solid State Phys. 13, 4855 (1980)

[7] T. Suski, J. Karpinski, K.L.I. Kobayashi and K.F. Komatsubara, J. Phys. Chem. Solids 42, $479(1981)$

[8] J. M.Leger and A. M. Redon, J. Phys. Condens. Matter 2, 5655 (1990)

[9] N. R. Serebryanaya, V. D. Blank, and V. A. Ivdenko, Phys. Lett. A. 197, 63 (1995)

[10] A. Onodera, I. Sakamoto, Y. Fujii, N. Mori, and S. Sugai, Phys. Rev. B 56, 7935 (1997)

[11] B. Kalkan, S. Sen, and S. M. Clark, J. Chem. Phys. 135, 124510 (2011) 
[12] K. M. Rabe and J. D. Joannopoulos, Phys. Rev. B 36, 3319 (1987)

[13] M. Hong, J. Zhou and Z.-G. Chen, Adv. Mater. 31, 1807071 (2019)

[14] A. Ciucivara, B. R. Sahu, and L. Kleinman, Phys. Rev. B 73, 214105 (2006)

[15] G. S. Do, J. Kim, S. H. Jhi, C. H. Park,S. G. Louie, and M. L. Cohen, Phys. Rev. B 82, $054121(2010)$

[16] Z. Sun,J. Zhou, H. K.Mao, and R. Ahuja, Proc. Natl. Acad. Sci. 109, 5984 (2012)

[17] S. Lee, K. Esfarjani, T. Luo, J. Zhou, Z. Tian and G. Chen, Nat. Commun. 5, 3525 (2014)

[18] M. Wuttig, V. L. Deringer, X. Gonze, C. Bichara, and J.-Y. Raty, Adv. Mater. 30, 1803777 (2018)

[19] J.-Y. Raty, M. Schumacher, P. Golub, V. L. Deringer, C. Gatti and M. Wuttig, Adv. Mater. 31, $1806280(2019)$

[20] J. C. Chervin, B. Canny, J. M. Besson, and P. Pruzan, Rev. Sci. Instrum. 66, 2595 (1995)

[21] B. Couzinet, N. Dahan, G. Hamel, and J. C. Chervin, High Press. Res. 23, 409 (2003)

[22] J. C. Chervin, B. Canny, and M. Mancinelli, High Press. Res. 21, 305 (2002)

[23] J. D. Barnett, S. Block, and G. J. Piermarini, Rev. Sci. Instrum. 44, 1 (1973)

[24] U. D. Wdowik, K. Parlinski, S. Rols, and T. Chatterji, Phys. Rev. B 89, 224306 (2014)

[25] M. Wuttig, D. Lüsebrink, D. Wamwangi, W. Welnic, M. Gilleßen, R. Dronskowski, Nat. Mater. 6, $122(2007)$

[26] D. Campi, L. Paulatto,G. Fugallo, F. Mauri, and M. Bernasconi, Phys. Rev. B 95, 024311 (2017)

[27] D. H. Dangic, A. R. Murphy, E. D. Murray, S. Fahy, and I. Savic, Phys. Rev. B 97, 224106 (2018)

[28] E. Steigmeier and G. Harbeke, Solid State Commun. 8, 1275 (1970)

[29] K. Jeong, S. Park,D. Park, M. Ahn, J. Han, W. Yang, H. S. Jeong, and M. H. Cho, Sci. Rep. 7, $955(2017)$

[30] J. W. Park, M. Song, S. Yoon, H. Lim, D. S. Jeong, B. K. Cheong, and H. Lee, Phys. Status Solidi A 210, 267 (2013)

[31] X. Zhou, Y. Du, J. K. Behera, L. Wu, Z. Song, and R. E. Simpson, ACS Appl. Mater. Interfaces. 8, 20185 (2016)

[32] P. Giannozzi, S. Baroni, N. Bonini, M. Calandra, R. Car,C. Cavazzoni,D. Ceresoli,G. L. Chiarotti, M. Cococcioni,I. Dabo, and A. Dal Corso, J. Phys. Condens. Matter 39, 395502 
(2009)

[33] P. Giannozzi, O. Andreussi, T. Brumme, O. Bunau, M. B. Nardelli, M. Calandra, R. Car, C. Cavazzoni, D. Ceresoli, M. Cococcioni, and N. Colonna, J. Phys. Condens. Matter 46, 465901 (2017)

[34] D. R. Hamann, Phys. Rev. B 88, 085117 (2013)

[35] M. Schlipf and F. Gygi, Comput. Phys. Commun. 196, 36 (2015)

[36] J. P. Perdew, K. Burke, and M. Ernzerhof, Phys. Rev. Lett. 77, 3865 (1996)

[37] S. Grimme, J. Comp. Chem. 27, 1787 (2006) 